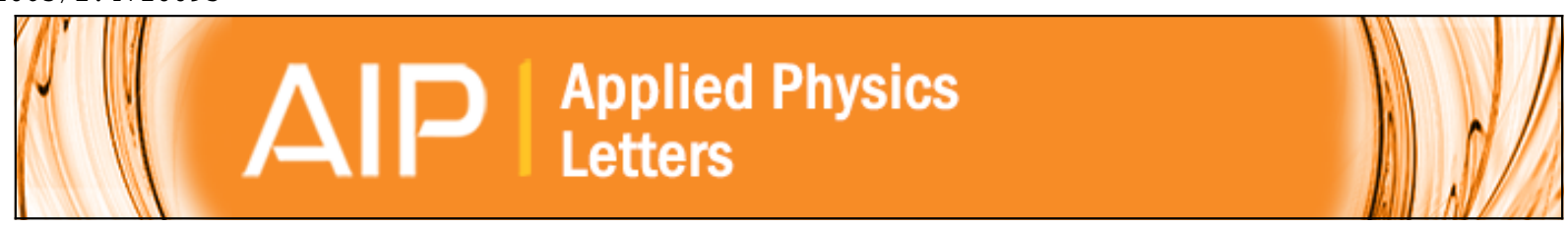

\title{
Tunable magnetoelectric response of dimensionally gradient laminate composites
}

C.-S. Park, D. Avirovik, M. I. Bichurin, V. M. Petrov, and S. Priya

Citation: Applied Physics Letters 100, 212901 (2012); doi: 10.1063/1.4720095

View online: http://dx.doi.org/10.1063/1.4720095

View Table of Contents: http://scitation.aip.org/content/aip/journal/apl/100/21?ver=pdfcov

Published by the AIP Publishing

\section{AlP}




\title{
Tunable magnetoelectric response of dimensionally gradient laminate composites
}

\author{
C.-S. Park, ${ }^{1}$ D. Avirovik, ${ }^{1}$ M. I. Bichurin, ${ }^{2}$ V. M. Petrov, ${ }^{2}$ and S. Priya ${ }^{1, a)}$ \\ ${ }^{1}$ Center for Energy Harvesting Materials and Systems (CEHMS), Virginia Tech, Blacksburg, \\ Virginia 24061, USA \\ ${ }^{2}$ Institute for Electronic and Information Systems, B. S. Peterburgskaya Street 41, \\ Veliky Novgorod 173003, Russia
}

(Received 19 April 2012; accepted 1 May 2012; published online 21 May 2012)

\begin{abstract}
A magnetoelectric (ME) sensor exhibiting wideband behavior as a function of applied magnetic DC bias and frequency was designed by combining the dimensionally gradient piezoelectric layer with Metglas magnetostrictive layers in laminate configuration. The ME coefficient of the band in the DC magnetic range of 52-242 Oe was measured to be $3000 \mathrm{mV} / \mathrm{cm}$ Oe under the resonant condition of $f=107 \mathrm{kHz}$. The wideband in the $\mathrm{AC}$ magnetic field frequency range of $41-110 \mathrm{kHz}$ had the ME coefficient in the vicinity of $260 \mathrm{mV} / \mathrm{cm}$ Oe under the conditions of $\mathrm{H}_{\mathrm{AC}}=1 \mathrm{Oe}$ and $\mathrm{H}_{\mathrm{DC}}=70 \mathrm{Oe}$. This frequency-dependent $\mathrm{ME}$ behavior clearly showed two different states on each side of the resonance peak which could open the possibility of developing new applications such as magnetic field-controlled switches.
\end{abstract}

(C) 2012 American Institute of Physics. [http://dx.doi.org/10.1063/1.4720095]

Composites with high magnitude of magnetoelectric (ME) coefficient have been projected to find applications in sensors, transducers, actuators, resonators, harvesters, and servomechanism. ${ }^{1-6}$ It is well-known that electromechanical resonance (EMR) in ME composites can be utilized to improve the magnitude of ME coefficent. $^{7-10}$ The EMR range can be tuned by designing the piezoelectric layers with gradient geometry. ${ }^{9} 10$ Gradient laminate composites in the vicinity of EMR can provide wideband behavior with high ME coefficient. Building upon this premise, we designed a ME resonator that exhibits flat and high ME response over wide magnetic DC bias and frequency range. In this study, we demonstrate that this design can provide tunable states as a function of frequency.

Dimensionally gradient piezoelectric plate ${ }^{9}$ with thickness of $1 \mathrm{~mm}$ was fabricated by mechanical polishing and dicing technique, as shown in Fig. 1(a). Piezoelectric plates with composition $\mathrm{Pb}\left(\mathrm{Zn}_{1 / 3} \mathrm{Nb}_{2 / 3}\right)_{0.2}\left(\mathrm{Zr}_{0.5} \mathrm{Ti}_{0.5}\right)_{0.8} \mathrm{O}_{3}$ (PZNT) were synthesized by conventional mixed oxide method. ${ }^{11}$ Piezoelectric constant of poled PZNT plates was found to be $500 \mathrm{pC} / \mathrm{N}$, and the dielectric constant was 2219 at $1 \mathrm{kHz}$. The piezoelectric voltage constant $\left(g_{33}\right)$ was of the order of $23.41 \times 10^{-3} \mathrm{Vm} / \mathrm{N}$. On this PZNT plate, $25 \mu \mathrm{m}$-thick Metglas (2605SA1, Metglas Inc, USA) sheets of desired dimensions were attached using epoxy (West System, USA) with the curing temperature of $80^{\circ} \mathrm{C}$. Finite element modeling software ATILA was used to model the structure and obtain resonance modes. ${ }^{9}{ }^{912}$ Impedance spectrum of the composites was measured by LCR meter (HP 4194 A). For ME voltage coefficient measurement in longitudinal-transversal (L-T) mode configuration, an electromagnet was used to apply the DC magnetic field, and the samples were placed in the center of the Helmholtz coil under an $\mathrm{AC}$ magnetic field $\left(\mathrm{H}_{\mathrm{ac}}\right)$. The voltage induced on the laminate was monitored by using a

\footnotetext{
${ }^{\text {a) }}$ Author to whom correspondence should be addressed. Electronic mail: spriya@vt.edu.
}

lock-in amplifier. The magnetostriction was evaluated by using the strain gauge and Wheatstone bridge.

Impedance spectrum of asymmetric electric plate was measured to identify the EMR range. In conjunction, ATILA simulation was conducted to observe the displacement behavior of $\mathrm{H}$-shaped piezoelectric plate as shown in Fig. 1(b). From simulation, the first, second, and third resonances were computed to be 42,44 , and $94.3 \mathrm{kHz}$, respectively. In comparison, measured impedance spectrums for the asymmetric $\mathbf{H}$-shaped piezoelectric plate exhibited resonance peaks at 42,44 , and $96 \mathrm{kHz}$, as shown in Fig. 1(b). Thus, the results between simulation and measurement were in good agreement. The first mode at $42 \mathrm{kHz}$ was associated with biaxial bending of the large rectangular section and the intermediate rectangular section, while the second mode at $44 \mathrm{kHz}$ was related to the biaxial bending of the large rectangular section and the small rectangular section. The third mode at $99 \mathrm{kHz}$ came from the lateral displacement of the large rectangular section, as shown in Fig. 1(c).

Figure 2(a) shows the fabricated ME laminate composite based on Fig. 1(a). On top of the PZNT layer, four layers of Metglas with the area of $13 \times 7 \mathrm{~mm}^{2}$ were attached at the middle rectangular section (Section A), thirty layers of Metglas with area of $20 \times 7 \mathrm{~mm}^{2}$ were attached at the larger rectangular section (Section B), and five layers of Metglas with area of $7 \times 7 \mathrm{~mm}^{2}$ were attached at the intermediate rectangular section (Section C), as shown in Fig. 2(a). There are two variables which could be adjusted to achieve a wideband ME response. ${ }^{9,10}$ First, if the rectangular area of two sections in piezoelectric plate is different than the one with smaller number of Metglas layers will show higher ME coefficient. Second, if the numbers of Metglas layers are same, the rectangular section with the smaller area will show smaller ME coefficient. Thus, by adjusting the ratio of Metglas layers on various rectangular sections, a composite ME response with flat behavior can be obtained. 
Figure 2(b) shows the magnetostriction $\left(\mathrm{S}_{\mathrm{ij}}\right)$ and piezomagnetic $\left(\mathrm{q}_{\mathrm{ij}}\right)$ coefficient for varying dimensions and stack configurations of Metglas. In this figure, $\mathrm{S}_{11}$ corresponds to longitudinal in-plane magnetostriction parallel to $\mathrm{H}_{\mathrm{DC}}$ and $\mathrm{q}_{11}$ is the longitudinal in-plain piezomagnetic coefficient corresponding to the differential of $S_{11}$. The maximum in-plane magnetostriction was found to be $28 \mathrm{ppm}$ regardless of Metglas stack configurations and dimensions; however, the strain behavior was strongly dependent on the stack configuration and dimensions. Four layers of Metglas with the area of $13 \times 7 \mathrm{~mm}^{2}$, thirty layers of Metglas with area of $20 \times 7 \mathrm{~mm}^{2}$, and five layers of Metglas with area of $7 \times 7 \mathrm{~mm}^{2}$ showed piezomagnetic coefficient corresponding to $0.38,0.21$, and $0.16 \mathrm{ppm} / \mathrm{Oe}$ at 70,150 , and $204 \mathrm{Oe}$ of $\mathrm{H}_{\mathrm{DC}}$, respectively. These piezomagnetic behaviors will result in strong elastic coupling with the piezoelectric sections.

Figure 2(c) shows the measured ME response from the composite structure as a function of magnetic DC bias under the condition of $\mathrm{H}_{\mathrm{AC}}=1 \mathrm{Oe}$ at $f=1 \mathrm{kHz}$. The peak at $70 \mathrm{Oe}$ was associated with Section A. The second peak of the ME
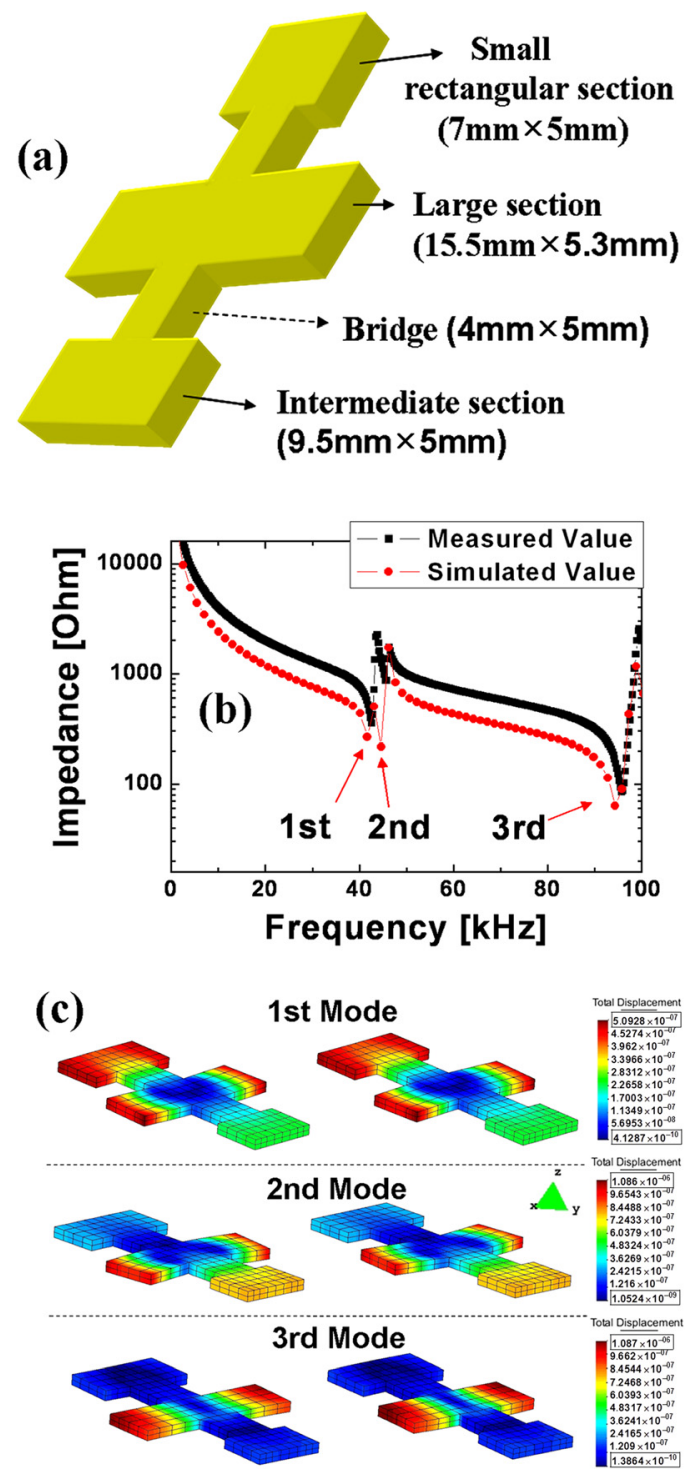

FIG. 1. (a) Schematic diagram of symmetric $\mathbf{H}$-shaped piezoelectric plate, (b) impedance spectrums between simulated and measured results, and (c) FEM analysis of resonant motions at 42, 44, and $94.3 \mathrm{kHz}$. coefficient at $150 \mathrm{Oe}$ was associated with Section C. The third peak of the ME coefficient at 209 Oe was associated with Section B. The value of DC bias at the peak in piezomagnetic coefficient corresponds to that for the peak in ME coefficient. Further, it was found that not only piezomagnetic coefficient but also piezoelectric dimensions affected elastic coupling between the Metglas and piezoelectric sections compared to Figs. 2(b) and 2(c). Furthermore, if only Section $\mathrm{A}$ and Section B were combined, there will be a valley in the intermediate range. By inserting Section $\mathrm{C}$, the formation of flat ME band was facilitated. The overall ME response as a function of magnetic DC bias was composite of three individual responses.

The impedance and phase angle spectrums for this laminate are shown in Fig. 3(a). After Metglas was attached on the PZNT plate, multiple resonances occurred at 14, 28, 41, 44, and $107 \mathrm{kHz}$ as marked with arrows in Fig. 3(a). Modal analysis conducted using ATILA is shown in Fig. 3(b). Resonant modes at 14 and $28 \mathrm{kHz}$ were found to be related to the bending vibrations of all the sections. The middle section dominated the vibration mode at $14 \mathrm{kHz}$ while the small
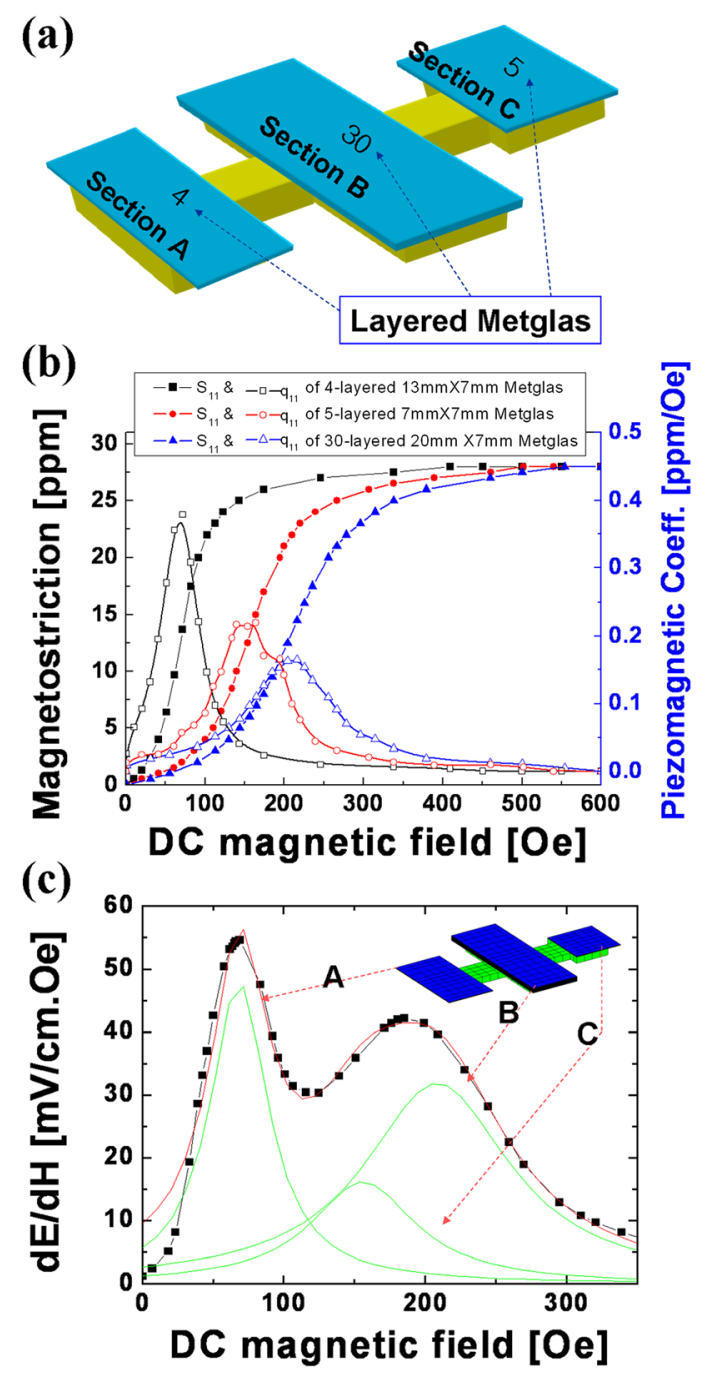

FIG. 2. (a) Schematic diagram of laminated ME composite, (b) magnetostriction $\left(\mathrm{S}_{11}\right)$ and piezomagnetic coefficient $\left(\mathrm{q}_{11}\right)$ for varying dimensions and stack configurations of Metglas, (c) ME response as a function of DC magnetic field under the constant condition of $\mathrm{H}_{\mathrm{ac}}=1 \mathrm{Oe}$ at $f=1 \mathrm{kHz}$. 

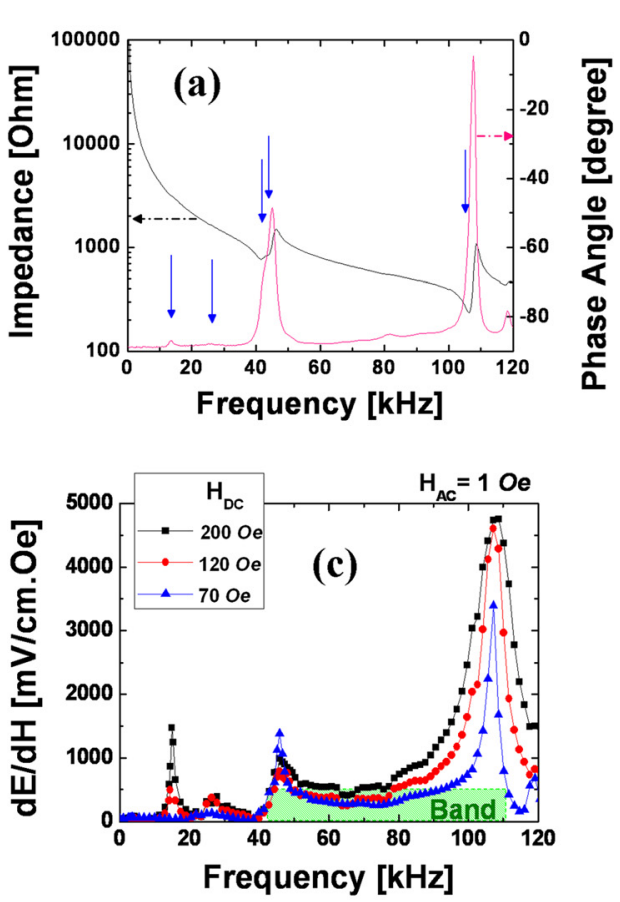
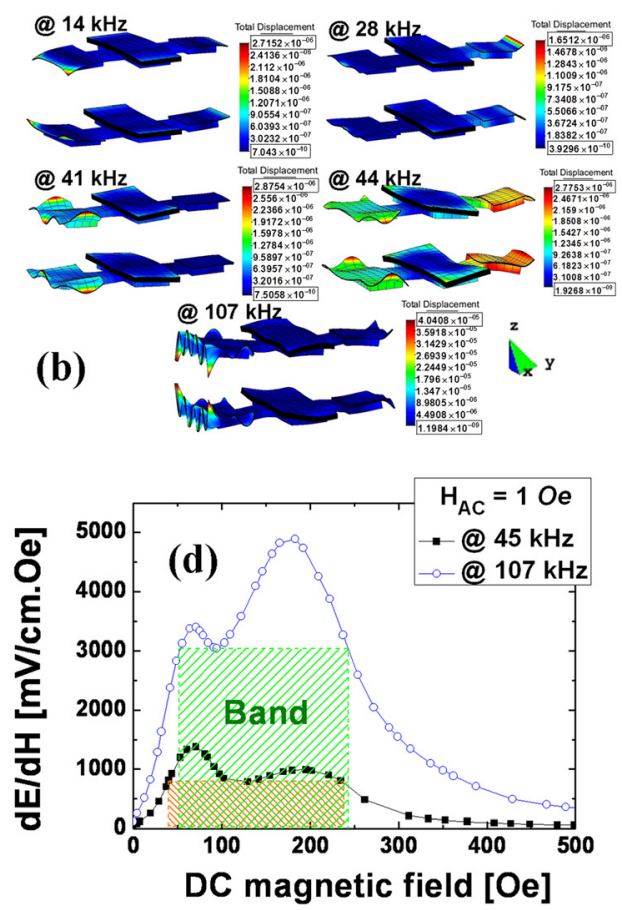

FIG. 3. (a) Impedance and phase angle spectrums of the ME composite, (b) resonant displacements at 14, 28, 41, 44, and $107 \mathrm{kHz}$ determined from simulations, (c) ME response as a function of frequency, (d) ME response as a function of DC magnetic field under resonance conditions at 45 and $107 \mathrm{kHz}$. rectangular section dominated the vibration mode at $28 \mathrm{kHz}$. The resonance at $41 \mathrm{kHz}$ was found to be combination of the bending motions of large rectangular section (Section B) and middle rectangular section (Section $\mathrm{A}$ ). The resonance at $44 \mathrm{kHz}$ was related to combined bending response from the bridge and small rectangular section (Section $\mathrm{C}$ ). The resonant modes of ME composite at 41 and $44 \mathrm{kHz}$ in Fig. 3(b) were correlated to the 1 st and 2 nd resonance modes of PZNT layer as determined in Fig. 1(c). The resonant mode at $107 \mathrm{kHz}$ had a similar vibration mode as that at $44 \mathrm{kHz}$.

The ME output voltage from composite was measured as a function of frequency under constant $\mathrm{H}_{\mathrm{AC}}=1 \mathrm{Oe}$ but varying $\mathrm{H}_{\mathrm{DC}}=70,120$, and $200 \mathrm{Oe}$, as shown in Fig. 3(c). Interestingly, $\mathrm{ME}$ response as a function of frequency had similar behavior as that of phase angle shift shown in Figs. 3(a) and 3(c). The ME peaks are shown at 14, 28, 45, and $107 \mathrm{kHz}$ corresponding to the resonance frequencies observed in the impedance measurement. The peak $\mathrm{ME}$ response at $45 \mathrm{kHz}$ was associated with resonances at 41 and $44 \mathrm{kHz}$. The maximum ME coefficient was found to be $4740 \mathrm{mV} / \mathrm{cm}$ Oe at $f=107 \mathrm{kHz}$ under $\mathrm{H}_{\mathrm{DC}}=200$ Oe. Interestingly, the bands were successfully formed in the range of $41-110 \mathrm{kHz}$. The composite showed high ME coefficient of $260 \mathrm{mV} / \mathrm{cm}$ Oe after the resonance peak of $41 \mathrm{kHz}$, regardless of the applied DC magnetic field. These widely extended bands were derived from the combination of the resonances at 41,44 , and $107 \mathrm{kHz}$.

The ME behavior at 45 and $107 \mathrm{kHz}$ as a function of DC magnetic field is shown in Fig. 3(d). The maximum ME coefficients of 1400 and $4740 \mathrm{mV} / \mathrm{cm} \mathrm{Oe}$ was found at 45 and $107 \mathrm{kHz}$, respectively. The band at $107 \mathrm{kHz}$ exhibited ME coefficient higher than $3000 \mathrm{mV} / \mathrm{cm}$ Oe from 52 to $242 \mathrm{Oe}$ of $\mathrm{H}_{\mathrm{DC}}$ while the band at $45 \mathrm{kHz}$ exhibited ME coefficient higher than $780 \mathrm{mV} / \mathrm{cm}$ Oe from 40 to 230 Oe of $\mathrm{H}_{\mathrm{DC}}$. The bands were obtained regardless of applied DC and AC magnetic fields. The wideband was formed in both conditions of the frequency range of $41-110 \mathrm{kHz}$ and DC mag- netic ranges of 40-230 Oe and 52-242 Oe at $f=45$ and $107 \mathrm{kHz}$, as shown in Figs. 3(c) and 3(d).

Interestingly, there are two noticeable states in the ME frequency response in Fig. 3(c). Under the constant DC magnetic field condition of $70 \mathrm{Oe}$, the composite showed flat ME responses in both frequency ranges of $1-11 \mathrm{kHz}$ (State I) and $41-110 \mathrm{kHz}$ (State II) as shown in Fig. 4. This figure also shows the schematic of resulting ME response as a function of frequency and the applied DC magnetic field conditions. State I shows a band lower than $129 \mathrm{mV} / \mathrm{cm} \mathrm{Oe} \mathrm{before}$ $f=41 \mathrm{kHz}$ while State II shows a band higher than $260 \mathrm{mV} /$ cm Oe after $f=41 \mathrm{kHz}$ under the constant condition of $\mathrm{H}_{\mathrm{DC}}=70 \mathrm{Oe}$. The ME coefficient of State II was $2 \times$ higher than that of State I. Consequently, $\Delta \mathrm{ME}\left(=\mathrm{ME}_{\text {StateII }}-\mathrm{ME}_{\text {StateI }}\right)$ was $131 \mathrm{mV} / \mathrm{cm}$ Oe under the constant $\mathrm{H}_{\mathrm{DC}}=70 \mathrm{Oe}$. These states were found to be adjustable with changing $\mathrm{H}_{\mathrm{DC}}$ conditions. The maximum value of the $\mathrm{ME}_{\mathrm{StateI}}$ can be reduced by decreasing the DC magnetic field and ranges between 0 and $129 \mathrm{mV} / \mathrm{cm}$ Oe. On the other hand, the minimum value of the

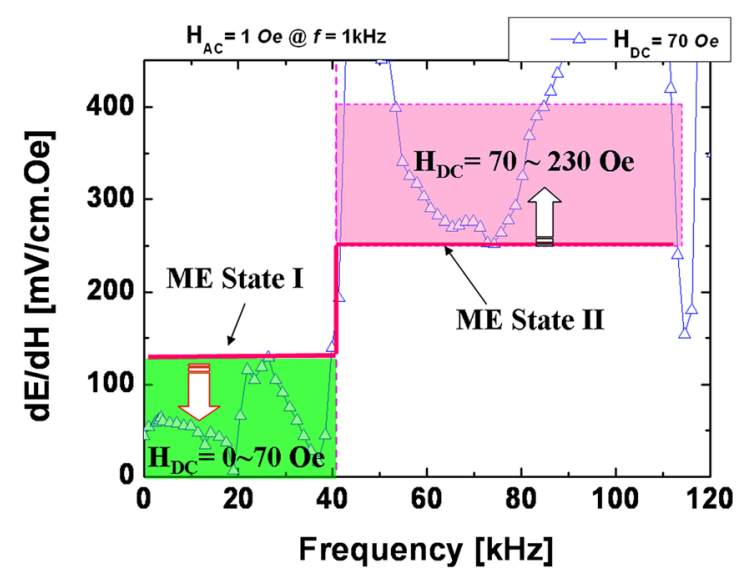

FIG. 4. Schematic of switchable ME response dependency on the frequency and DC magnetic field conditions. 
$\mathrm{ME}_{\text {StateII }}$ can be elevated by increasing DC magnetic field condition in the range of $260-406 \mathrm{mV} / \mathrm{cm}$ Oe. Thus by tuning the magnitude of frequency and $\mathrm{H}_{\mathrm{DC}}, \Delta \mathrm{ME}$ in the range of $131-406 \mathrm{mV} / \mathrm{cm}$ Oe can be achieved which clearly shows the tunability of this device. These signals are strong enough to allow two distinguishable states. The clear sensing margin was seen between State I and State II: the ME values in State II were at least two times higher than that in State I. This interesting and promising ME behavior can be exploited in several applications. The wideband behavior can be a candidate for magnetic field controlled switches as well as ME harvesters. In the case of the magnetic field-controlled switches, the resonant frequency becomes the cut-off condition and the State I and State II can be considered as "off" and "on," respectively.

Next, we attempt to explain the reason for the formation of switching states by calculating the in-plane strain and stress components for all sections and at the end combining them together to find the overall solution for mode formation. The total vibration spectrum of the laminate composite consists of several contributions. The first mode is associated with simultaneous bending vibrations of the small rectangular section with its bridge and the middle rectangular section. ${ }^{13}$ The equation of bending motion of $i$ th-area ( 1 and 2 areas correspond to bridge and section) has the form:

$$
\nabla^{2} \nabla^{2} w_{i}+\frac{\rho_{i} t_{i}}{D_{i}} \frac{\partial^{2} w_{i}}{\partial t^{2}}=0
$$

where $\nabla^{2} \nabla^{2}$ is biharmonic operator, $w_{i}$ is the displacement in z-direction, $t_{i}$ is thickness, $\rho_{i}$ is average density of $i$-area, and $D_{i}$ is cylindrical stiffness. The strain's components can be expressed in terms of displacement as $S_{1 i}=-z \frac{\partial^{2} w_{i}}{\partial x^{2}}$ and $S_{2 i}=-z \frac{\partial^{2} w_{i}}{\partial y^{2}}$. The stress components can be expressed in terms of strains as

$$
\begin{aligned}
& \left({ }^{p} S_{k}\right)_{i}={ }^{p} S_{k j}\left({ }^{p} T_{j}\right)_{i}+{ }^{p} d_{31}{ }^{p} d_{3 i}, \\
& \left({ }^{m} S_{k}\right)_{i}={ }^{m} S_{k j}\left({ }^{m} T_{j}\right)_{i}+\left({ }^{m} g_{k 1}\right)_{i}{ }^{m} S_{1 i},
\end{aligned}
$$

where $S_{1 i}$ and $T_{1 i}$ are strain and stress components for $i$-area, $E_{3 i}$ is the component of electric field, $H_{1 i}$ is the component of magnetic field, $s_{k j}$ is compliance at constant electric field for piezoelectric and at constant magnetic induction for magnetic component, $g_{k 1}$ and $d_{31}$ piezomagnetic and piezoelectric coefficients, correspondingly. The superscripts $p$ and $m$ correspond to piezoelectric and piezomagnetic layers. Solving Eq. (1) for displacement of each section by using the boundary conditions given below provides the dynamic solution

$$
w_{1}=0 \text { and } \partial w_{1} / \partial x=0 \text { at } x=0,
$$

$w_{1}=w_{2}, \partial w_{1} / \partial x=\partial w_{2} / \partial x,\left(M_{1}\right)_{1}=\left(M_{1}\right)_{2}$, and $\left(V_{1}\right)_{1}=\left(V_{1}\right)_{2}$ on the boundary of 1 and 2 areas;

$\left(M_{1}\right)_{2}=0$ and $\left(V_{1}\right)_{2}=0$ at $x=L(L$ is the total length of section and bridge);

$\left(M_{2}\right)_{i}=0$ and $\left(V_{2}\right)_{i}=0$ at $y= \pm b_{i} / 2\left(b_{i}\right.$ is the width of $i$-area),

where $\left(M_{j}\right)_{i}$ is the moment of rotation and $\left(V_{j}\right)_{i}$ is the transverse force with respect to $\mathrm{j}$-axis.

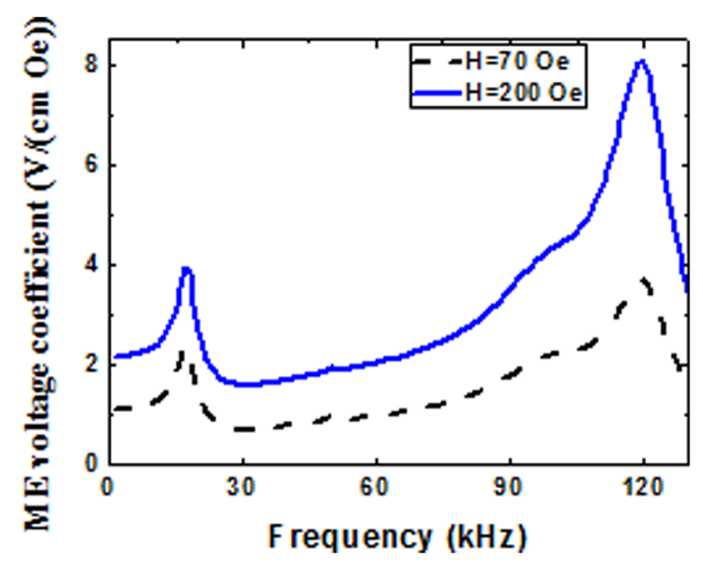

FIG. 5. Frequency dependence of ME voltage coefficient for MetglasPZNT composite for bias field of 200 and 70 Oe.

The computed displacements were used to determine the strain components and then the stress components from Eq. (2). Substituting the stress components into open circuit condition enables the calculation of the ME voltage coefficient by taking into account condition that average electric field induced across the piezoelectric layer is estimated as integral of internal electric field taken over the piezoelectric thickness, $E=\frac{1}{t_{p}} \int_{t_{p}}{ }^{p} E_{3} d z$. These calculations were carried out numerically. The resonance frequencies for the small rectangular section and the middle rectangular section were found to be approximately equal. The second harmonic of this mode can be seen at $97 \mathrm{kHz}$. It should be noted that theoretical estimate for the next resonance frequency (at $44 \mathrm{kHz}$ ) as the bending mode of large rectangular section was made using similar procedure. The bridge was not included into vibrating system since its displacement (in $\mathrm{z}$ direction) was negligible. Finally, the mode at $120 \mathrm{kHz}$ was calculated under assumption that it came from the axial mode of the large rectangular section. The estimate for this case was found using the computing method known heretofore. ${ }^{4}$ Figure 5 clearly reveals that frequency band of $90-130 \mathrm{kHz}$ arises from the combination of the resonance modes. These states are adjustable with $\mathrm{H}_{\mathrm{DC}}$.

In summary, we demonstrate a ME resonator exhibiting wideband behavior by fabricating a dimensionally gradient structure and combining with laminate configuration. We were able to obtain a flat ME response in the DC magnetic bias range of 52-242 Oe where the ME coefficient was higher than $3000 \mathrm{mV} / \mathrm{cm}$ Oe under resonant condition. The promising wideband behavior as a function of frequency occurred in the range of $41-110 \mathrm{kHz}$ where the ME coefficient was higher than $260 \mathrm{mV} / \mathrm{cm}$ Oe independent of applied $\mathrm{H}_{\mathrm{DC}}$. Under low $\mathrm{H}_{\mathrm{DC}}$ condition, two different ME states were clearly obtained.

This work was financially supported by Office of Basic Energy Science, Department of Energy, USA (DE-FG0208ER46484).

${ }^{1}$ T. Kimura, T. Goto, H. Shintani, K. Ishizaka, T. Arima, and Y. Tokura, Nature (London) 426, 55 (2003).

${ }^{2}$ N. A. Spaldin and M. Fiebig, Science 309, 391 (2005).

${ }^{3}$ J. Ryu, A. V. Carazo, K. Uchino, and H.-E. Kim, J. Electroceram. 7, 17 (2001).

${ }^{4}$ C.-W. Nan, M. I. Bichurin, S. Dong, D. Viehland, and G. Srinivasan, J. Appl. Phys. 103, 031101 (2008). 
${ }^{5}$ G. Srinivasan, Annu. Rev. Mater. Res. 40, 153 (2010).

${ }^{6}$ J. Ma, J. Hu, Z. Li, and C.-W. Nan, Adv. Mater. 23, 1062 (2011).

${ }^{7}$ D. V. Chashin, Y. K. Fetisov, K. E. Kamentsev, and G. Srinivasan, Appl. Phys. Lett. 92, 102511 (2008).

${ }^{8}$ S. Dong, J. Zhai, J. Li, and D. Viehland, Appl. Phys. Lett. 89, 252904 (2006).

${ }^{9}$ C.-S. Park, C.-W. Ahn, J. Ryu, W.-H. Yoon, D.-S. Park, H.-E. Kim, and S. Priya, J. Appl. Phys. 105, 094111 (2009).
${ }^{10}$ C.-S. Park, C.-W. Ahn, S.-C. Yang, and S. Priya, J. Appl. Phys. 106, 114101 (2009).

${ }^{11}$ S.-M. Lee, C.-B. Yoon, S.-H. Lee, and H.-E. Kim, J. Mater. Res. 19, 2553 (2004).

${ }^{12}$ C.-S. Park, D. Avirovik, S. Bressers, and S. Priya, Appl. Phys. Lett. 98, 062904 (2011).

${ }^{13}$ V. M. Petrov, G. Srinivasan, M. I. Bichurin, and T. A. Galkina, J. Appl. Phys. 105, 063911 (2009). 